\title{
Biophysical Process
}

National Cancer Institute

\section{Source}

National Cancer Institute. Biophysical Process. NCI Thesaurus. Code C19986.

Affecting biological factors or systems, a Biophysical Process is a subatomic, atomic, or molecular process that involves passive, physical movement; attraction or repulsion (electrostatic, van der Waals, gradient, hydrogen bonding, hydrophobic, hydrophilic, etc.); radiation interaction; or non-enzymatic formation of covalent bonds. 